\title{
Techniques of new application for acquisition foreign languages: development stages and modern trend
}

\author{
Imad Tahini ${ }^{1, *}$, and Aliaksei Dadykin ${ }^{1}$ \\ ${ }^{1}$ Belarusian National Technical University, Department of Information Systems and Technologies, 220056, 50 let Pobedy, 11-38, Minsk, \\ Belarus
}

\begin{abstract}
In the conditions of scientific and technical progress and more developing international contacts in the field of science, technology, and culture, the practical knowledge of a foreign language becomes one of the most important characteristics of a highly qualified specialist of any profile. One of the important factors in the development of the system to accelerating learning is the demands of adults themselves. Therefore, we need to create an automated system fast and effective teaching another language that uses the mini-max criterion, which provides the maximum possible result to the minimum required amount of effort on the part of the student. The essence of the approach is to replace the description in our native language with visual objects using the latest technology for implementing this approach. This means that an intermediate language is introduced, not tied to any of the known languages. The Purpose of the research is to develop a methodology (technique) for applying the Visual Approach in the educational environment created on the basis of a distributed management system to accelerate the acquisition of foreign language skills by adults. The system provides the process of controlling the formation of speech skills to a threshold level, allowing the transition from language learning to its improvement and acceleration. On the basis of this new generation using continuous evaluation of the competency level of the trainee, visual models, and information technology, got them a new incarnation, opens a new direction in construction a new version of Learning Management System - E-AMS.
\end{abstract}

\section{Introduction}

Modern life is simply impossible without knowledge of a foreign language, foreign language culture. Development of creative abilities, creating conditions for selfexpression and self-realization of Adults is one of the most important areas of modern language education. This will be accelerated by new effective teaching and learning foreign languages methods which integrates the framework of a holistic approach and computer technology. The aim of our project is to develop such a methodology. This will help both young people and older people to accumulate knowledge and acquire skills that will enable them to become more independent and responsible in judgments and actions and be open in relationships with others in the global community.

Traditional training programmes and institutions are quite inefficient and very costly to meet these challenges. In addition, unlike schoolchildren and young students, adult education requires a special personalized approach, taking into account the current level of their language skills, general education, work and professional skills, personal and family situation, origin, general cultural background and other factors. In fact, each student needs to make a personal curriculum and constantly adjust it based on their results, successes and failures, systematic and regular classes, and many other factors, up to the daily routine and the load of household and industrial activities. Actually it is impossible to provide high-quality and personalized programs in manual mode. We consider automated learning management systems to be the best solution available.

At the same time, the existing LMS are mainly aimed at the management of standard educational processes for the transfer of theoretical knowledge in the education system and are not suitable for monitoring and managing the processes of acquiring primary language and professional skills. The task of providing a training system with necessary content for different countries, languages, activities and professions is really complicated and requires a lot of time and resources.

Therefore, it is necessary to create a new generation of tools that provide not only the structuring and transfer of knowledge from teacher to student, but also quantitative and qualitative management of the level of skills and competencies of the student and the parameters of their real progress along the learning curve. This is especially important in the field of adult education and retraining. As it was shown in the works of Bandura [1], learning occurs in the process of interaction of the external environment, human behaviour and personal properties which influence each other greatly.

\footnotetext{
* Corresponding author: imad.tahini@live.com
} 
In this article, we propose a concept of building a new generation of systems for the formation of language skills of foreign language proficiency for adults using the proposed Structural-Visual Method (SVM) [2, 3], which is based on scientific theories from various branches of knowledge to facilitate the learning in a more efficient way and offer personalized learning.

\section{Development of language skills using E-AMS}

New generations of teachers and students are searching for new effective and timesaving methods and techniques to use in teaching. Unfortunately, traditional teaching techniques don't meet learners' and teachers' expectations, since the formal speech approach does not provide real conditions for the formation of psychophysiological mechanisms that ensure the competent use of the language.

\subsection{Proposed structural visual model}

Structural-visual method in linguistics is a representation of the structure of information of the subject industry and linguistic knowledge in graphical form using color to encode the most common patterns (Figure.1). Thus obtained visual models replace text explanations (rules) in the formation of appropriate skills and abilities. Visual symbols and visual properties of model elements (shape, size, relative position, frames, font, underscores, etc.) are used to encode other important properties and parameters of system elements. This approach in linguistics allows to transform information about the structure of the language from verbal to visual form, and thus eliminate the shortcomings of the grammatical approach and construct proposed new effective system E-AMS.

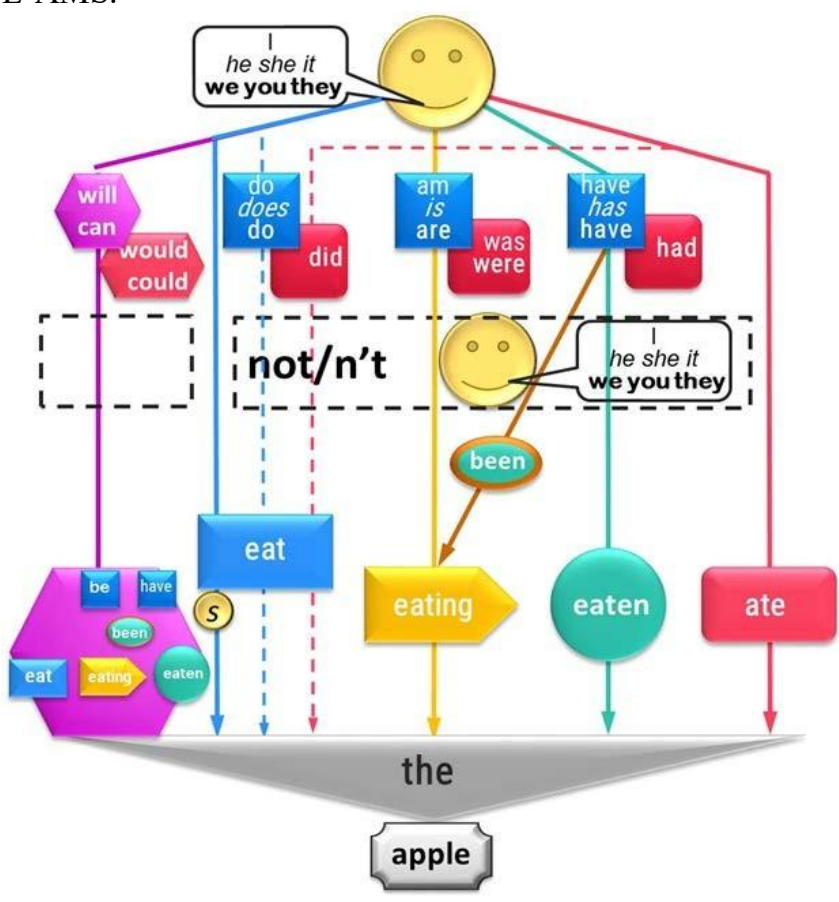

Fig. 1. Full model of all active Levels.
This method helps to understand the grammatical worldview of English speakers. In just one session of "Anglo-psychotherapy" you get a holistic view of the dynamics of English grammar. And this is important for an adult. Of course, this does not exclude the need for intensive training. But now it becomes clear what to train! In addition, today the author has a unique grammar training system [2].

The main problem arising in the study of English grammar, it is that a person thinks and analyzes in the same place in the brain where he speaks. However, a person is not physiologically able to simultaneously speak and analyse. These two processes are mutually exclusive - one can do either one or the other. As a result, when try to speak and think in grammars rules, we can less freely to be fluently speaking a foreign language. So we need new techniques that allow the trainer to learn how to build correct English sentences without textbooks and complex grammatical rules, and speak fluently, without hesitation and painful thoughts.

This technique replaces:

-From Difficult terms and rules to simple and clear maps-diagrams.

-From abstract theory to effective training.

-From Boring texts to simple actions in real situations.

The prepared materials that contain educational program contents of (SVM) have been tested on group of students and got positive results [3] like other training methods of Galperin theory [4].

\subsection{Process of elimination of the delay during learning}

In Figure 2 shows a simplified diagram of the interaction of speakers of different languages within the same subject area. For example, if the work on a joint project in the IT sphere is carried out by specialists from different countries, their linguistic interaction should be considered not in the field of the entire language space, but only in the part that relates to their professional activities. Similarly, it makes no sense to train refugees for a long time in all the intricacies of a new language, it is necessary to give them the language skills necessary for a new profession as soon as possible and to ensure open and direct communication within this subject area.

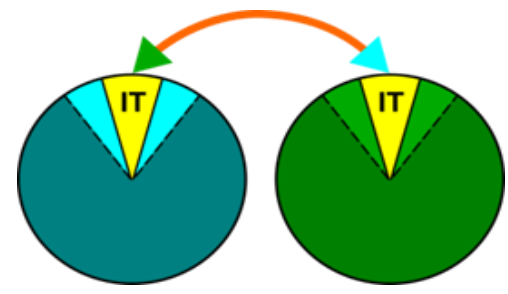

Fig. 2. Interaction between native speakers of different languages within the same subject area.

The main form of communication is the transmission of the necessary meaning through dialogue. The minimum possible fragment of the dialogue (Figure 3) is an elementary Question, the simplest Answer and the Delay between them. In case of a conversation between partners speaking different languages, an extra delay $\Delta$ is 
inevitably added to the process of their dialogue, related to the internal translation of the information received from the language of the question to its presentation in their minds in their native language. The same process occurs in the opposite direction when forming the answer.

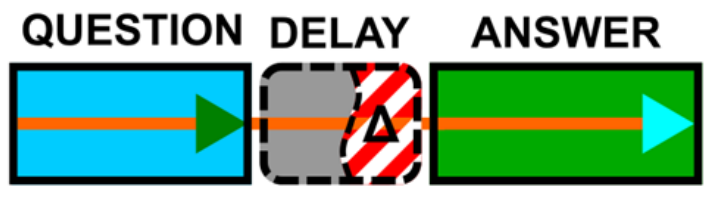

Fig. 3. A fragment of dialogue between native speakers of different languages.

Considering the same processes from the point of view of training, or rather developing language skills in a foreign language, we can safely say that either the teacher or any training system should strive to compensate as quickly as possible for this internal delay $\Delta \rightarrow 0$, then the language zone of the new language will be formed in the consciousness of the learner, or in other words, the curve of learning a foreign language will reach the point of "spontaneous speaking". That is, the construction of the ideal process of learning a foreign language is reduced to the creation of such learning tools that guarantee the complete elimination of the delay $\Delta=0$ in the shortest possible time.

\subsection{Transition to the language competency}

The process of forgetting information is fast enough, but Electronic Acquiring Management System (E-AMS) will ensure the formation of a logarithmic dependence of the learning curve, will avoid the "barrier of overcoming" and prevent the transition of training in the mode of retraining (Figure 4).

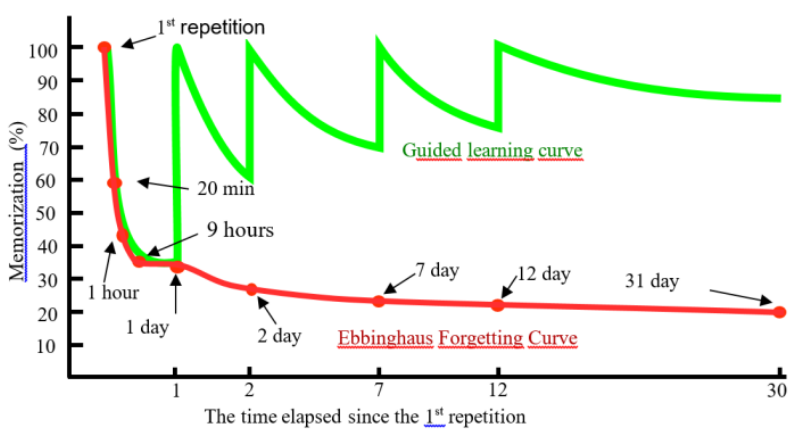

Fig. 4. Comparison of Guided and Unguided Learning Processes.

The proposed E-AMS is aimed primarily at the formation of listening and speaking skills, which are fundamental $[5,6,7]$ and make up about $75 \%$ of the total volume of necessary competencies (Figure 5). As we can see, written speech makes up less than $10 \%$ of the total language competence, so it is not the goal to set and reach in our system. In addition, there are several variants of perfectly working Internet systems that are specifically designed to study grammar in writing, and can be easily added to the proposed E-AMS.

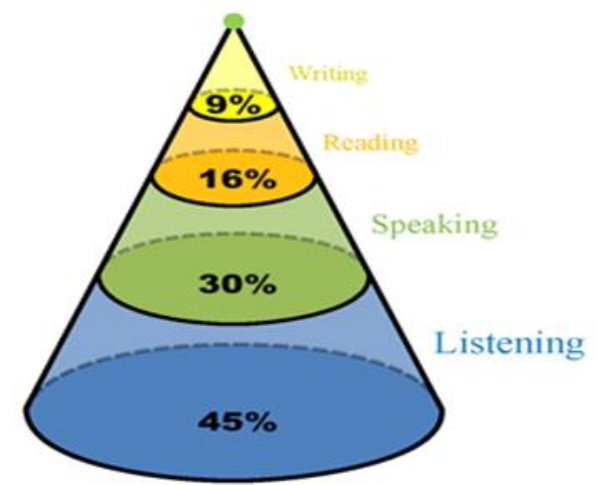

Fig. 5. Distribution of language competences.

In fact, the description of the process of obtaining the necessary result is reduced to mathematical dependence: $\mathrm{Rez}=$ Fminmax $(\mathrm{Li}, \mathrm{Sp}, \mathrm{Re}, \mathrm{Wr}) \approx 350$ hours,

where: $\mathrm{Li}$ - listening, $\mathrm{Sp}$ - speaking, $\mathrm{Re}$ - reading, $\mathrm{Wr}$ writing.

Moreover, particular importance is attached to the correlation between R_(Li+Sp), R_(Li+Re) and $\mathrm{R} \_(\mathrm{Li}+\mathrm{Wr})$, because the process of developing language skills is not determined by simple trial, and works only if the learner seeks as accurately as possible to imitate the sounds of the new language.

Therefore, typical lessons in E-AMS (Figure 6) lasting 25-30 minutes each should consist of:

- Pre-recorded introductory part (3-5 minutes), in which the teacher in the native language of the student explains the content of the lesson,

- Main part (about 20 minutes) which can be carried out in a virtual classroom for the chosen profession and uses the main components of the SVM - Visual Dictionary and Visual Models,

- Final part (5-7 minutes) is a grammatical training based on maps-tables, perfecting the language skills up to the required level,

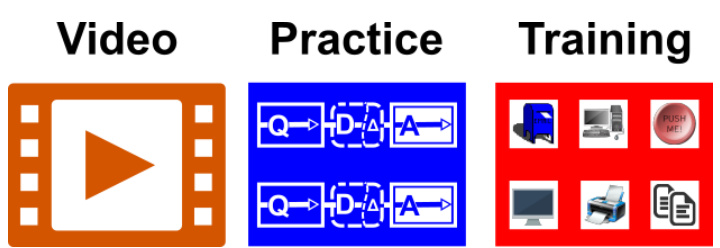

Fig. 6. Schematic diagram of an E-AMS typical lesson structure.

\subsection{Display the formation of training skills}

This learning approach helps to develop foreign language communication skills in the shortest possible time along with simultaneous development of the grammatical system of the language. Only in this way foreign language thinking can be formed quickly. In this case, learners begin to express their thoughts competently and freely, it is easier to perceive the speech of people around them and gradually become an active participant in the communication process. As a result, we can see not only coexistence and contact of the old and the new language in the mind of the learner, but also the direct influence of the experience of the native language on an accurate perception of reality in another 
language, the direct interpenetration of naturally digestible and consciously learned language material, which is particularly important when teaching adults. As experience shows, unsystematic mastering of a foreign language is extremely unproductive.

In addition, it should be well understood that the main contradiction that prevents the creation of effective tools for rapid mastery of another language is the logical closure between the language as a tool for managing activities and the language as a subject of activity. The essence of this problem is that if the student does not know how to make a proposal that conveys the desired meaning, then he will not be able to do it. But if he knows the rules how to do it, then he is still not able to do it, since the area of the brain responsible for speaking is busy thinking about this rule.

\begin{tabular}{|c|c|c|c|c|c|c|c|c|c|c|c|c|c|}
\hline \multirow{2}{*}{$\begin{array}{l}\text { CEFR } \\
\text { Levels }\end{array}$} & \multirow{2}{*}{$\begin{array}{c}\text { Cambridge } \\
\text { Exams }\end{array}$} & \multicolumn{12}{|c|}{ Months } \\
\hline & & 1 & 2 & 3 & 4 & 5 & 6 & 7 & 8 & 9 & 10 & 11 & 12 \\
\hline C2 & CPE & & & & & & & & & & $t=$ & & \\
\hline C1 & CAE & & & & & $a=$ & & & & $F$ & 163 & 177 & $\begin{array}{c}191 \\
1\end{array}$ \\
\hline B2 & FCE & & & & & & & 111 & 126 & 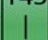 & 116 & 164 & \\
\hline & & & & & & & 94 & 1 & 112 & 127 & & & \\
\hline B1 & PET & & & 5 & & 13 & 78 & 95 & & & & & \\
\hline & & & & & 60 & 61 & & & & & & & \\
\hline$A 2$ & KET & $=$ & 의 & 40 & 1 & & & & & & & & \\
\hline A1 & & & 24 & $\begin{array}{c}1 \\
25\end{array}$ & & & & & & & & & \\
\hline at & Lessons & $\begin{array}{l}1 \\
1\end{array}$ & 10 & & & & & & & & & & \\
\hline
\end{tabular}

Fig. 7. Approximate order of use of Visual Models in accordance with the learning curve.

Thus, the development of a new language is a transition to a new way of thinking, causing speech production in accordance with the grammatical schemes and semantic connections of the system of the studied language. It is here that the epistemological roots of the proposed SVM are located (see Figure 7).

The proposed approach makes it possible to form a speech zone on the basis of learning not separate words and grammar, but to use typical situations, dialogues, short sentences, collocations, speech patterns and even slang. A General view of the process of formation of a new speech zone is shown in the Figure 8 .

Teaching materials should be presented in about 90 video tutorials and about 60 training exercises. The corresponding Common European Framework of Reference (CEFR) test system is quite suitable as control tests when moving from one level to another.

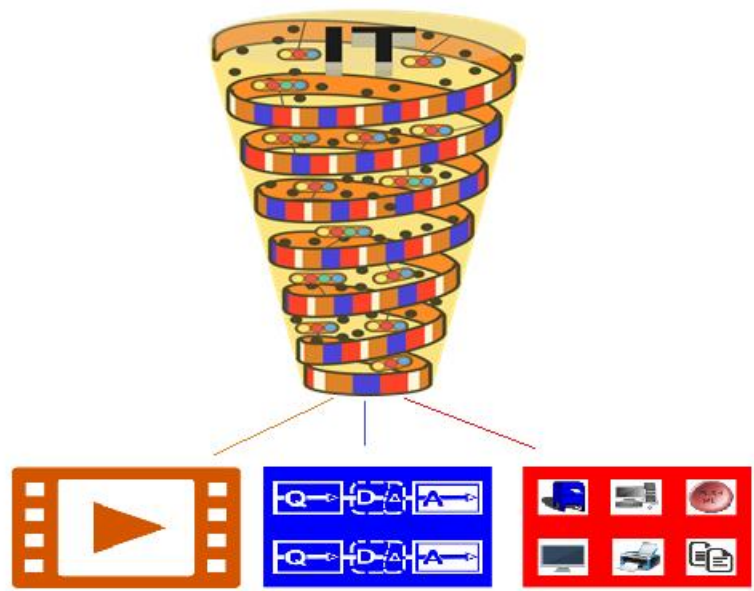

Fig. 8. General view of a new speech zone formation process in EAMS.

During the work of the system, a detailed statistical analysis of the results is carried out, dynamic learning curves of each adult learner are displayed, tables of coefficients are substantiated and indicators of the speed of speech skills formation are specified, determine the levels of speaking from primary to spontaneous speaking are determined in accordance with the scale of CEFR. Figure 9 displays the authors' ideas mentioned above.

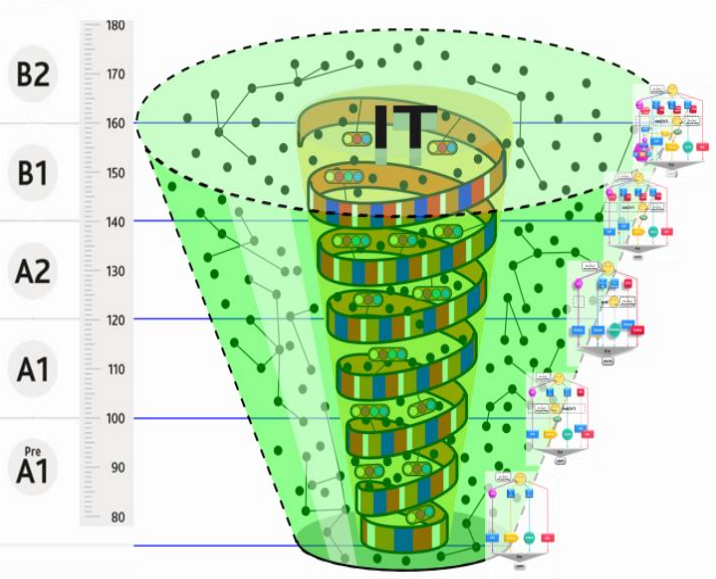

Fig. 9. Visual representation E-AMS.

\section{Structure of electronic management system}

Now the conceptual solution has been found. Innovative training system allowing to operate the process of developing professional and language skills of adults has been created and it combines the following:

- Methodological principles, grounded in the works of Bandura and Galperin [4],

- Structural Visual Method [8] that forms a synergetic effect, both in the initial phase of foreign language learning, and at the stage of "overcoming the barrier",

- Using the achievements of the ICT sphere as a tool to ensure the implementation of learning objectives with 
continuous monitoring of the current state and obtaining a guaranteed learning result in a finite number of steps.

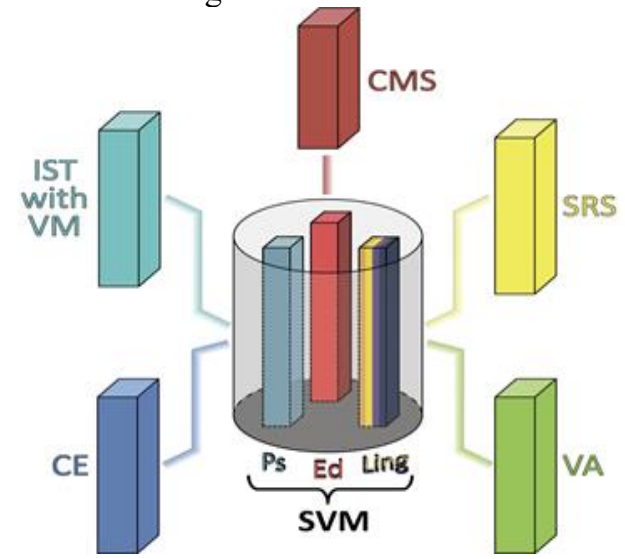

Fig. 10. E-AMS generalized structure.

The generalized structure of E-AMS is a distributed control system and consists of (Figure 10):

- Interactive Speech Trainers (IST) that use visual vocabulary and Visual Models of the language being acquired [9];

- Content Management System (CMS),

- Speech Recognition and modeling System (SRS),

- System of Continuous Evaluation (CE) of the current language competence in combination with Virtual Assistant (VA).

Currently, the development of the main components of the E-AMS is carried out in four interrelated areas:

- Further improvement of the visual approach modeling the structure of the mastered activity on the basis of interactive visual dictionary and visual models,

- Creation of a set of interactive speech simulators with elements of augmented reality, video lessons and training exercises corresponding to different levels of students' competence,

- Setting up a system of continuous evaluation and management of the learning process in real time.

- CMS development in the Content Management System, repositories of training materials for video lessons and mechanisms for managing the synthesis of training exercises are formed.

The last two directions of development of EAMS are connected exclusively with Big Data. The language system is not limitless and, in fact, is quite observable, but its full use presents numerous opportunities for combining, which until recently exceeded all possible system conditions, and only now the use of Big Data technology can successfully solve these problems.

In turn, the system of continuous evaluation and management of the educational process provides the configuration of the entire system at three main levels:

- Interface of speech simulators, where all the achievements in the field of gamification, socialization and cooperation should be fully provided for the translation of the educational process into a modern, intensive and effective format,

- Teacher, where the current statistical analysis of the results of the language activity of each student is carried out,
- Continuous measurement and management of the learning process at each specific point of the learning curve or retraining in real time, which provides an individual approach to the parameters of a particular skill training, as the speed of the exercises is not set by external instructions or the interface of the training program, but by the abilities, capabilities and level of competence of each individual student.

Currently, the first prototypes of interactive trainers using visual models have been created. To implement the full functioning of the AMS, 3-7 standard simulators are required at each of the increasing levels of language proficiency in accordance with the program of developing learners' professional. The total number of trainers is about 25.

E-AMS is created on a modular basis, so each element of the system can be effectively used separately. At the same time, the synergetic effect of the joint use of all elements of the system and the integration of additional developments can significantly exceed the capabilities of existing analogues and will help to solve very important social problems, including the problems of training in professional skills and language adaptation of refugees and migrants.

\section{Development and Implementation}

The program is based on a unique technique that allows you to learn the language in the way that adults do intuitively, without dictionaries and grammar reference books. Such an approach may scare off the adherents of the "old school". As you stay in which you will not only learn, but "absorb" the language. This is facilitated by a large set of interactive lessons, the purpose of which is to create an associative series. This new system is known as (Lingvomap) is suitable primarily for adults. Using a microphone, the program can evaluate the correctness of your pronunciation. Another important point for Lingvomap is a gradual change and complication of goals and objectives, according to as users acquire new skills and competencies. Main aspects:

- Dynamic interface: use of map and images requiring attention user and real-time response.

- Interaction: use techniques that provide a connection and let the user to interact with the application through voice commands.

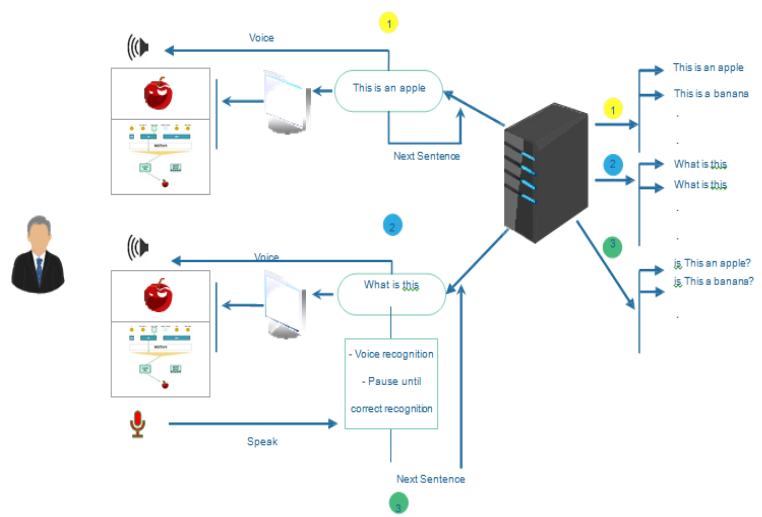

Fig. 11. Functional diagram of the system. 
This solution allows monitoring and control of a student's learning activities in the following ways (Figure 11):

- User chooses the lesson.

-Then the sentences of a lesson will display in sequential steps.

-Each sentence will be displayed as image and map, and then the system speaks the sentence.

-User will interact with each sentence for a lesson by view the map that represent the construction of sentence and action image of this sentence.

- User tries to speak correct sentence in target language.

-The program can evaluate the correctness of user pronunciation and save the result in database.

-At the end of lesson the statistical result will be displayed.

In order to implement such application, we should go in the development stages to reach optimal solution and open new horizon about learning, currently we are building this platform, and the development started in implementing the client - server architecture [10], server side contain the schema of the database that contains interrelated tables to design the repository of data upon the analysis the situation of the foreign language to construct data lesson with its levels, so each level of lessons will be formulated to contain set of sentences and index of each map and action image, The database under development and experiment and every time it will be expended to get full view of system design, as we still in initial stage we will still do the improvement and change the database diagram to get real situation and get full process of data flow for the courses under the request from functions that responsible to prepare CMS , The main focus of CMS is educational content to provide authors, designers, and experts with the means to more effectively to create educational materials. The main task solved by CMS is to create the required content over a specific time period to meet the needs of individual students, by reducing the basic grammatical structures (Figure. 12), we can create a set of required words and create the structure of sentence of a target training lesson, Template is the interrelation of the structure and language to create Data for a lesson [10].

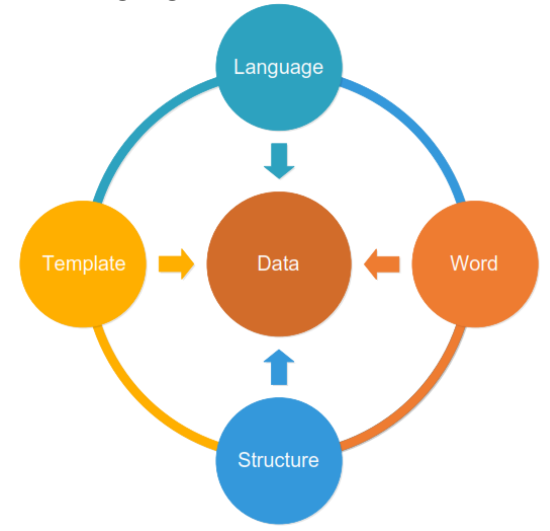

Fig. 12. Generate of Data Lesson.

The design of the interface of this application will be located at client side to display the working of the program, the development code of this program is going in parallel by using web development and creates mobile android application (Figure 13), Till now several web simulators are created and tested.

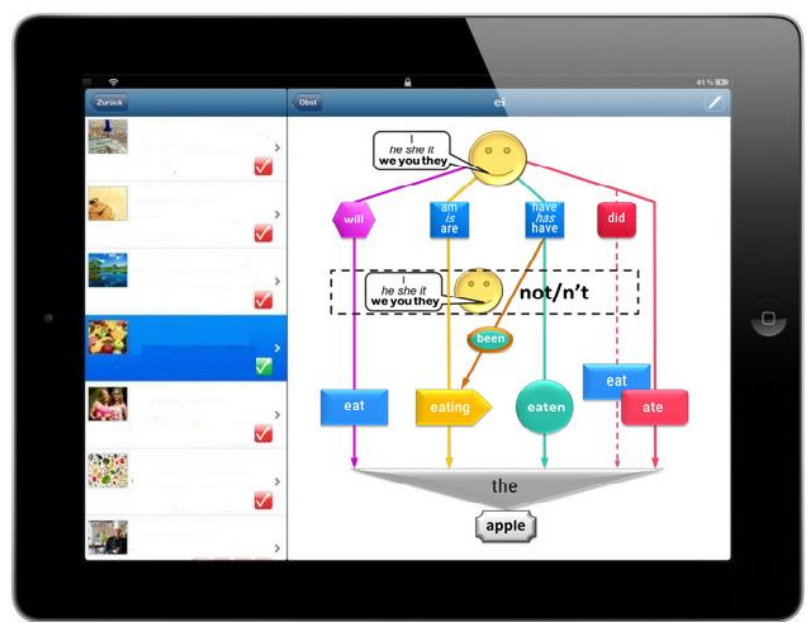

Fig. 13. Interface of the system in mobile application.

\section{Conclusion}

The conceptual solution is based on the use of mechanisms of system analysis and unification of scientific concepts of representatives of the Soviet school and scientists of Western countries against the background of the technological leap of the second decade of the 21 st century. Using visual tools to analyze abstract systems and theories allows you to discover new patterns and simplifies their understanding. The use of visual models as a guide for practical training has a long history and positive implementation experience in many areas. This project is a joint project of researchers from the Lebanon, Belarus, United States, Japan and Ukraine. The authors of the project emphasize that it does not contradict the existing system of assessment of language competencies in Europe, but rather contributes to their importance. The project is planned in accordance with the priorities of the European Commission (topic: Addressing the challenge of migrant integration through ICT-enabled solutions) under the Horizon 2020 program. Currently, the work is underway to implement this approach in educational applications, programs and a new type of E-AMS.

\section{References}

1. A. Bandura \& R.H. Walters, (1963). Social learning and personality development(New York: Holt, Rinehart, \& Winston)

2. A.K. Dadykin, V.A. Dibrova and I.H. Tahini, The Visual Approach in Educational Projects, International Journal of Social Science and Humanity. Vol. 7, No. 6, June 2017, pp. 373-377

3. Boyd L. Bradbury, Imad H. Tahini, Alex K. Dadykin, Fundamentals of New Effective System to Accelerate Language Acquisition Using Visual Approach, International Journal of Information and 
Education Technology, Vol. 8, No. 11, November 2018, pp. 768-772

4. P.Y. Galperin, Psychology of thinking and teaching about the gradual formation of mental actions, Research in the thinking of Soviet psychology (Moscow, 1966)

5. Wilt, Miriam E, A study of teacher awareness of listening as a factor in elementary education, Journal of Educational Research, 43 (8), April, 1950, pp. 626-636

6. British Council [Electronic resource], Five essential listening skills for English learners By Raphael Ahmed. - 2015. - Mode of access: https://www.britishcouncil.org/voicesmagazine/five-essential-listening-skills-englishlearners. - Date of access: 30.11.2018

7. University of Missouri Extension [Electronic resource], Listening: Our Most Used Communications Skill. - Mode of access: https://extension2.missouri.edu/cm150. - Date of access: 30.11 .2018

8. I.H. Tahini, T. Nakayama, V.A.Dibrova, and A.K. Dadykin, Cognitive Psychology Models and Approaches to Develop Language Skills, International Journal of Social Science and Humanity. Vol. 8, No. 4, April 2018, pp. 110-115

9. I.H. Tahini, A.K. Dadykin, V.A. Dibrova, Control System of Foreign Language Training Based on Structural-Visual Techniques, In Proceedings 4th International Conference on Computer Science Computer Engineering and Education Technologies (Beirut, Lebanon, April 2017, pp.149-154)

10. Imad H. Tahini, Alex K. Dadykin, Proposed System of New Generation LMS Using Visual Models to Accelerate Language Acquisition. Advances in Science, Technology and Engineering Systems Journal, Vol. 3, No. 5, October 2018, pp. 277-287 